\title{
Gravitational waves in vacuum spacetimes with cosmological constant. I. Classification and geometrical properties of non-twisting type $N$ solutions.
}

\author{
Jiří Bičák and Jiří Podolský \\ Department of Theoretical Physics, Faculty of Mathematics and Physics, \\ Charles University, V Holešovičkách 2, 18000 Prague 8, Czech Republic \\ Electronic addresses: bicak@mbox.troja.mff.cuni.cz,podolsky@mbox.troja.mff.cuni.cz
}

\begin{abstract}
All non-twisting Petrov-type $N$ solutions of vacuum Einstein field equations with cosmological constant $\Lambda$ are summarized. They are shown to belong either to the non-expanding Kundt class or to the expanding Robinson-Trautman class. Invariant subclasses of each class are defined and the corresponding metrics are given explicitly in suitable canonical coordinates. Relations between the subclasses and their geometrical properties are analyzed. In the subsequent paper these solutions are interpreted as exact gravitational waves propagating in de Sitter or anti-de Sitter spacetimes.
\end{abstract}

PACS number(s): 04.30.-w, 04.20.Jb, 98.80.Hw 


\section{INTRODUCTION AND SUMMARY}

The purpose of this and the subsequent paper is to analyze all non-twisting type $N$ solutions of Einstein's vacuum equations with $\Lambda$. There are several basic works on these solutions available in literature, in particular [1]-[3] (for pre-1980 works, see Ref. [4]). None of them, however, discusses the physical interpretation of the solutions. Such an interpretation, based on the study of the deviation of geodesics, will be presented in the following paper. In this part we summarize, compare, classify and generalize the mathematical results of Refs. [1]-[3].

We consider type $N$ solutions in which the Debever-Penrose null vector field $\mathbf{k}$ is quadruple 四. The vector field defines a congruence of null geodesics $x^{\alpha}(v)$ such that $d x^{\alpha} / d v=k^{\alpha}, k_{\alpha} k^{\alpha}=0, k_{\alpha ; \beta} k^{\beta}=0, v$ being an affine parameter. In general a geodesic congruence is characterized by its expansion $\Theta=\frac{1}{2} k_{; \alpha}^{\alpha}$, shear $|\sigma|=\sqrt{\frac{1}{2} k_{(\alpha ; \beta)} k^{\alpha ; \beta}-\Theta^{2}}$

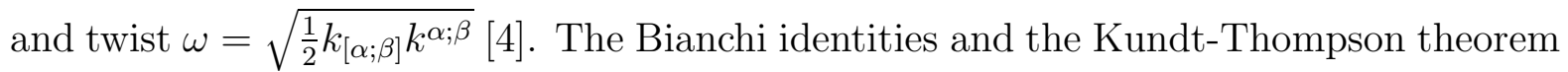
for type $N$ solutions (see [5], Theorem 7.5 in [4]) imply $\sigma=0$ since $C_{\alpha \beta \gamma \delta} ; \delta=R_{\alpha \beta \gamma \delta} ; \delta=0$ for solutions of $R_{\alpha \beta}=\Lambda g_{\alpha \beta}$. In the following we assume $\omega=0$. Therefore, with (possibly) non-vanishing $\Lambda$ we are left with two cases to consider: (i) the Kundt class of non-expanding gravitational waves, $\Theta=0$ (cf. [6], Ch.27 in [4]), and (ii) the RobinsonTrautman class of expanding gravitational waves of type $N, \Theta \neq 0$ (cf. [7], Ch.24 in [4]). Hereafter we denote the Kundt class by $K N(\Lambda)$ and the Robinson-Trautman class by $R T N(\Lambda)$.

Section II analyzes the class $K N(\Lambda)$ in detail. We do not present any new solution of this type but we extend the results of Ref. [3] by giving the explicit forms of the transformations which leave the metrics of $K N(\Lambda)$ invariant. These enable us to give the explicit transformations to different canonical subclasses of $K N(\Lambda)$, which have not been given in literature so far. We also introduce a convenient new notation for these subclasses and demonstrate how they are interconnected. In particular, we show that one of the subclasses is identical to the 'Lobatchewski waves' studied by Siklos [8]. We also formulate the proposition (proven in Appendix) that all vacuum solutions with $\Lambda$ which are conformal to the 'Kundt waves with $\Lambda=0$ ' belong to one specific subclass of $K N(\Lambda)$; we thus generalize the result of an analogous theorem for the $p p$-waves $[8]$. 
In Section III the Robinson-Trautman solutions [7] of type $N$ with $\Lambda$ are discussed. The transformation between two standard forms of the metric (those of Refs. [4] and [1]) is presented, and then transformations preserving the metric form given in [1] are generalized and used to demonstrate how the non-radiative part of the structural function of these solutions can be transformed away.

\section{THE KUNDT CLASS OF SOLUTIONS $K N(\Lambda)$}

This class has been investigated in detail by Ozsváth, Robinson and Rózga [3]. They have shown that in this case the vector $\mathbf{k}$ can be normalized such that $\frac{1}{2} \mathcal{L}_{\mathbf{k}} g_{\alpha \beta}=\frac{1}{2}\left(k_{\alpha ; \beta}+\right.$ $\left.k_{\beta ; \alpha}\right)=L k_{\alpha} k_{\beta}$, where $\mathcal{L}_{\mathbf{k}}$ is the Lie derivative and $L$ is a scalar. Denoting $L^{\prime}=\mathcal{L}_{\mathbf{k}} L$, we find $\mathcal{L}_{\mathbf{k}} L^{\prime}=0$ so that $L^{\prime}$ is invariant under renormalization of $\mathbf{k}$. A suitable coordinate system $(v, \xi, \bar{\xi}, u)$, where $\xi, \bar{\xi}$ are space-like coordinates, $v$ is a parameter along the null geodesics and $u$ is a retarded time with $u=$ const. being a wavefront, $\mathbf{k} \equiv \psi(u) \partial_{v}$, can be introduced [3] in which the $K N(\Lambda)$ metrics have the form

$$
d s^{2}=2 \frac{1}{p^{2}} d \xi d \bar{\xi}-2 \frac{q^{2}}{p^{2}} d u d v+F d u^{2}
$$

where

$$
\begin{aligned}
& p=1+\frac{\Lambda}{6} \xi \bar{\xi}, \quad q=\left(1-\frac{\Lambda}{6} \xi \bar{\xi}\right) \alpha+\bar{\beta} \xi+\beta \bar{\xi}, \\
& F=\kappa \frac{q^{2}}{p^{2}} v^{2}-\frac{\left(q^{2}\right),_{u}}{p^{2}} v-\frac{q}{p} H, \quad \kappa=\frac{\Lambda}{3} \alpha^{2}+2 \beta \bar{\beta} .
\end{aligned}
$$

Here $\alpha(u)$ and $\beta(u)$ are arbitrary real and complex functions of $u$, respectively. These functions play the role of two arbitrary 'parameters', i.e., we can denote the Kundt class by $K N(\Lambda) \equiv K N(\Lambda)[\alpha, \beta]$. The parameter $\kappa$ is related to the invariant $L^{\prime}$ by

$$
L^{\prime}=\kappa \frac{p^{2}}{q^{2}} .
$$

The function $H=H(\xi, \bar{\xi}, u)$ entering $F$ is restricted by Einstein's equations, $H_{, \xi \bar{\xi}}+$ $\left(\Lambda / 3 p^{2}\right) H=0$. There exists a general solution to this equation

$$
H(\xi, \bar{\xi}, u)=\left(f_{, \xi}+\bar{f}_{, \bar{\xi}}\right)-\frac{\Lambda}{3 p}(\bar{\xi} f+\xi \bar{f})
$$


where $f(\xi, u)$ is an arbitrary function of $\xi$ and $u$, analytic in $\xi$. The space-time is conformally flat if and only if the structural function $H$ is of the form

$$
H=H_{c}=\frac{1}{p}\left[\left(1-\frac{\Lambda}{6} \xi \bar{\xi}\right) \mathcal{A}+\overline{\mathcal{B}} \xi+\mathcal{B} \bar{\xi}\right],
$$

with $\mathcal{A}(u)$ and $\mathcal{B}(u)$ being arbitrary real and complex functions, respectively. Since $H_{c}$ of this form corresponds to (3) for $f$ quadratic in $\xi$ we easily infer the following

Lemma 1: The $K N(\Lambda)$ solutions (11), (3) with $f=f_{c}=c_{0}(u)+c_{1}(u) \xi+c_{2}(u) \xi^{2}$, where $c_{i}(u)$ are arbitrary complex functions of $u$, are isometric to Minkowski (if $\Lambda=0$ ), de Sitter $(\Lambda>0)$ and anti-de Sitter spacetime $(\Lambda<0)$.

It can be proven by straightforward but tedious calculations that the following lemma (which is not formulated in [3] but is a consequence of results therein) is true:

Lemma 2: The metric of the $K N(\Lambda)[\alpha, \beta]$ class preserves its form (1) under the transformations $(v, \xi, \bar{\xi}, u) \rightarrow(w, \eta, \bar{\eta}, t)$ given by

$$
\begin{aligned}
& v=a(t)\left[w+\frac{\left(1-\frac{\Lambda}{6} \eta \bar{\eta}\right) \gamma+\delta \bar{\eta}+\bar{\delta} \eta}{\left(1-\frac{\Lambda}{6} \eta \bar{\eta}\right) \alpha^{\prime}+\beta^{\prime} \bar{\eta}+\bar{\beta}^{\prime} \eta}\right]+\Delta(t), \\
& \xi=\frac{\bar{B}(t)+A(t) \eta}{\bar{A}(t)-\frac{\Lambda}{6} B(t) \eta}, \quad u=u(t),
\end{aligned}
$$

where $A(t), B(t)$ are arbitrary complex and $a(t), u(t)$ are real functions of $t$, respectively.

In the new coordinates $(w, \eta, \bar{\eta}, t)$ the resulting metric $K N(\Lambda)\left[\alpha^{\prime}, \beta^{\prime}\right]$ has

$$
\begin{aligned}
& \alpha^{\prime}=\frac{\sqrt{a \dot{u}}}{\Phi}\left[\left(A \bar{A}-\frac{\Lambda}{6} B \bar{B}\right) \alpha+\bar{A} B \beta+A \bar{B} \bar{\beta}\right], \\
& \beta^{\prime}=\frac{\sqrt{a \dot{u}}}{\Phi}\left[-\frac{\Lambda}{3} \bar{A} \bar{B} \alpha+\bar{A}^{2} \beta-\frac{\Lambda}{6} \bar{B}^{2} \bar{\beta}\right],
\end{aligned}
$$

with $\Phi=A \bar{A}+\frac{\Lambda}{6} B \bar{B}$ and the dot denotes $d / d t$. The remaining real functions $\gamma(t), \Delta(t)$ and a complex function $\delta(t)$ in (5) must satisfy the equations

$$
\begin{aligned}
\alpha^{\prime} \delta-\beta^{\prime} \gamma & =\frac{1}{\Phi}(\bar{A} \dot{\bar{B}}-\dot{\bar{A}} \bar{B}) \equiv C, \\
\bar{\beta}^{\prime} \delta-\beta^{\prime} \bar{\delta} & =\frac{1}{\Phi}\left(\dot{A} \bar{A}-A \dot{\bar{A}}+\frac{\Lambda}{6} \dot{B} \bar{B}-\frac{\Lambda}{6} B \dot{\bar{B}}\right) \equiv D, \\
\frac{\kappa^{\prime}}{a} \Delta & =\frac{1}{2}\left(\frac{\dot{a}}{a}-\frac{\ddot{u}}{\dot{u}}\right)-\left(\frac{\Lambda}{3} \alpha^{\prime} \gamma+\bar{\beta}^{\prime} \delta+\beta^{\prime} \bar{\delta}\right) .
\end{aligned}
$$


The structural function $H^{\prime}(\eta, \bar{\eta}, t)$ then takes the form

$$
p^{\prime} H^{\prime}=\left(1-\frac{\Lambda}{6} \eta \bar{\eta}\right) E+\bar{F} \eta+F \bar{\eta}+\frac{\dot{u}}{\Phi} \sqrt{\frac{\dot{u}}{a}}\left(A-\frac{\Lambda}{6} \bar{B} \bar{\eta}\right)\left(\bar{A}-\frac{\Lambda}{6} B \eta\right) p H
$$

where

$$
\begin{aligned}
E(t) \equiv & 2 \dot{\gamma}+\frac{2}{a}\left(\alpha^{\prime} \Delta\right)^{\cdot}-\alpha^{\prime} \kappa^{\prime} \frac{\Delta^{2}}{a^{2}}-2 \frac{\Delta}{a}\left[\frac{1}{2} \alpha^{\prime}\left(\frac{\dot{a}}{a}+\frac{\ddot{u}}{\dot{u}}\right)+C \bar{\beta}^{\prime}+\bar{C} \beta^{\prime}\right] \\
& -\alpha^{\prime}\left(2 \delta \bar{\delta}-\frac{\Lambda}{3} \gamma^{2}\right)+2 \gamma\left(\beta^{\prime} \bar{\delta}+\bar{\beta}^{\prime} \delta\right), \\
F(t) \equiv & 2 \dot{\delta}+\frac{2}{a}\left(\beta^{\prime} \Delta\right)^{\cdot}-\beta^{\prime} \kappa^{\prime} \frac{\Delta^{2}}{a^{2}}-2 \frac{\Delta}{a}\left[\frac{1}{2} \beta^{\prime}\left(\frac{\dot{a}}{a}+\frac{\ddot{u}}{\dot{u}}\right)-\frac{\Lambda}{3} C \alpha^{\prime}-D \beta^{\prime}\right] \\
& -\frac{\Lambda}{3} \gamma\left(\beta^{\prime} \gamma-2 \alpha^{\prime} \delta\right)+2 \bar{\beta}^{\prime} \delta^{2} .
\end{aligned}
$$

The transformation (5) is also important in connection with Lemma 1. Comparing Eq. (4) with Eq. (8), we see that we can 'generate' $H=H_{c}$ from $H=0$ by a coordinate transformation. Therefore, the conformally flat part, $H_{c}$, of $H$ generated by $f_{c}$ cannot represent a radiative field. Only if the function $f$ is at least cubic in $\xi$, the resulting spacetime is of type $N$ and can be interpreted as radiative (see the subsequent paper).

Another important application of the coordinate freedom (5) was suggested in [3]: it is possible to use the transformation to get 'canonical' subclasses of $K N(\Lambda)[\alpha, \beta]$ corresponding to special values of parameters $\alpha$ and $\beta$. Without loss of generality we can assume $\alpha \geq 0$. In addition, the transformation (5) of a special form

$$
\xi=\eta, \quad v=a(t) w, \quad u=\int a(t) d t
$$

for $a(t) \neq 0$ results in scaling $\alpha(u)=\alpha^{\prime}(t) /|a(t)|, \beta(u)=\beta^{\prime}(t) /|a(t)|$ so that we can always assume either $\alpha=1$ or $\alpha=0$. Since the parameter $L^{\prime}$ (2) is invariant, the sign of $\kappa$ is also an invariant. We can thus base the invariant canonical classification of all $K N(\Lambda)$ solutions on the sign of $\kappa$ and the sign of $\Lambda$. There are nine possible cases $(\kappa$ and $\Lambda$ can both be positive, zero or negative). However, subclasses $\kappa<0, \Lambda>0$ and $\kappa=0, \Lambda>0$ and $\kappa<0, \Lambda=0$ are forbidden since they violate the relation $\kappa=\frac{\Lambda}{3} \alpha^{2}+2 \beta \bar{\beta}$. The remaining possibilities are six subclasses which we shall now discuss.

A. Subclass $\kappa=0, \Lambda=0$. The equation $\kappa=0$ implies $\beta=0$ so that $\alpha=1$. A canonical representative of this subclass can be denoted as $P P \equiv K N(\Lambda=0)[\alpha=1, \beta=0]$. We 
are now using the notation $P P$ since the corresponding metric

$$
P P: \quad d s^{2}=2 d \xi d \bar{\xi}-2 d u d v-(g+\bar{g}) d u^{2}
$$

with arbitrary $g(\xi, u)=f_{, \xi}$ analytic in $\xi$, describes well-known $p p$ waves investigated by many authors (for details and references see section 21.5 in 四).

B. Subclass $\kappa>0, \Lambda=0$. Since $0<\kappa=2 \beta \bar{\beta}$, we have $\beta \neq 0$. Then Eqs. (5) with $A=\sqrt{\beta}, B=\left(-\frac{1}{2} \frac{\alpha}{\beta}+i \frac{J}{\beta}\right) A, a=\frac{1}{\beta \beta}, \gamma=\frac{1}{2}\left(\frac{\alpha}{\sqrt{\beta \bar{\beta}}}\right)+\frac{2 J}{\alpha}\left(\frac{J}{\sqrt{\beta \bar{\beta}}}\right) \cdot \delta=\dot{A} / A, \Delta=\frac{1}{2} \dot{a}, u=t$, where $J$ is a real function satisfying $\left(\frac{J}{\sqrt{\beta \bar{\beta}}}\right)^{\cdot}=\frac{i}{4} \frac{\alpha}{\sqrt{\beta \bar{\beta}}} \frac{\bar{\beta}}{\beta}\left(\frac{\beta}{\beta}\right)^{\prime}$, transform any solution of this subclass to $K N \equiv K N(\Lambda=0)[\alpha=0, \beta=1]$. The metric

$$
K N: \quad d s^{2}=2 d \xi d \bar{\xi}+2(\xi+\bar{\xi})^{2}\left[-d v+\left(v^{2}-\frac{g+\bar{g}}{\xi+\bar{\xi}}\right) d u\right] d u
$$

with arbitrary $g(\xi, u)=\frac{1}{2} f_{, \xi}$, describes solutions discovered by Kundt [6]. This is a special 'Kundt solution' in the 'Kundt class' of non-expanding waves. For details see [4], Chapter 27; the transformation between $\tilde{v}$ used there and $v$ above is $v=\tilde{v} /(\xi+\bar{\xi})^{2}$.

C. Subclass $\kappa>0, \Lambda>0$. If $\alpha=1$ we can use (5) with $A=\exp \left(-\frac{i}{2} \chi\right), B=c A$, $a=\left(\frac{\Lambda}{6}+\beta \bar{\beta}\right)^{-1}, \quad \gamma=-\left[\dot{W}+\frac{1}{2}(\dot{V} \exp (-i \chi)+\dot{\bar{V}} \exp (i \chi))\right] /\left(1+\frac{\Lambda}{6} c \bar{c}\right), \quad \delta=\dot{A} / A+$ $\frac{\Lambda}{6} \dot{c} \bar{c} /\left(1+\frac{\Lambda}{6} c \bar{c}\right), \quad \Delta=\frac{1}{4} \dot{a}-\frac{\Lambda}{12}(c \bar{c})^{\cdot}\left(1+\frac{\Lambda}{6} c \bar{c}\right)^{-1} a, \quad u=t$, where $c \equiv V+W \bar{A} / A, \quad V \equiv \frac{6}{\Lambda} \bar{\beta}$, $W \equiv-\frac{6}{\Lambda} \sqrt{\frac{\Lambda}{6}+\beta \bar{\beta}}, \chi$ is a real function satisfying $\dot{\chi}=\frac{i}{2}(\dot{V} \exp (-i \chi)-\dot{\bar{V}} \exp (i \chi)) / W$ to obtain $\alpha^{\prime}=0, \beta^{\prime}=1$. If $\alpha=0$ the scaling (9) makes $|\beta|=1$ and then we put $\xi=\frac{6}{\Lambda} \beta / \eta$, $v=w+\frac{1}{2}(\dot{\beta} \bar{\beta} \eta+\beta \dot{\bar{\beta}} \bar{\eta}) /(\eta+\bar{\eta}), u=t$. In both cases the representative of this subclass is $K N(\Lambda) I \equiv K N(\Lambda)[\alpha=0, \beta=1]$. The metric reads

$K N(\Lambda) I$ :

$$
d s^{2}=2 \frac{d \xi d \bar{\xi}}{\left(1+\frac{\Lambda}{6} \xi \bar{\xi}\right)^{2}}-2\left(\frac{\xi+\bar{\xi}}{1+\frac{\Lambda}{6} \xi \bar{\xi}}\right)^{2} d u d v+\left[2\left(\frac{\xi+\bar{\xi}}{1+\frac{\Lambda}{6} \xi \bar{\xi}}\right)^{2} v^{2}-\frac{\xi+\bar{\xi}}{1+\frac{\Lambda}{6} \xi \bar{\xi}} H\right] d u^{2}
$$

$K N(\Lambda) I$ indicates that this solution is a generalization of the $K N$ waves to the case $\Lambda \neq 0$, ' $I$ ' means 'of the first kind'. The $K N(\Lambda) I$ solutions were first discovered by García Díaz and Plebański [1], [9], transformation between their coordinates and those used here is $\xi=\sqrt{\frac{6}{\Lambda}} \tanh \sqrt{\frac{\Lambda}{6}} \tilde{\xi}, v=r / \sinh \sqrt{\frac{\Lambda}{6}}(\tilde{\xi}+\overline{\tilde{\xi}}), u=-\sqrt{\frac{\Lambda}{6}} t$. However, in [1], [9] not all $K N(\Lambda \neq 0)$ solutions were found since (invariantly different) subclasses $\mathrm{E}$ and $\mathrm{F}$ mentioned below were omitted. 
D. Subclass $\kappa>0, \Lambda<0$. The same transformation as in the previous case leads to the metric (12) which has thus the same form for $\Lambda<0$. The transformation to the coordinates used in [1] is $\xi=\sqrt{-\frac{6}{\Lambda}} \tan \sqrt{-\frac{\Lambda}{6}} \tilde{\xi}, v=r / \sin \sqrt{-\frac{\Lambda}{6}}(\tilde{\xi}+\overline{\tilde{\xi}}), u=-\sqrt{-\frac{\Lambda}{6}} t$.

E. Subclass $\kappa<0, \Lambda<0$. Now $\kappa<0$ implies $\alpha=1$. Using (5) with $A=\exp (i \phi)$, $B=c A, a=\left(1+\frac{6}{\Lambda} \beta \bar{\beta}\right)^{-1}, \gamma=0, \delta=\bar{A}^{2} \dot{\bar{c}} /\left(1+\frac{\Lambda}{6} c \bar{c}\right), \Delta=\frac{3}{2 \Lambda} \dot{a}, u=t$, where $c \equiv$ $\frac{1}{\beta}\left(\sqrt{1+\frac{6}{\Lambda} \beta \bar{\beta}}-1\right), \phi$ is a real function satisfying $\dot{\phi}=i \frac{\Lambda}{12}(\dot{c} \bar{c}-c \dot{\bar{c}}) /\left(1+\frac{\Lambda}{6} c \bar{c}\right)$, we find $\beta^{\prime}=0$ and the canonical representative, denoted by $K N\left(\Lambda^{-}\right) I I \equiv K N(\Lambda<0)[\alpha=1, \beta=0]$, is $K N\left(\Lambda^{-}\right) I I:$

$$
d s^{2}=2 \frac{d \xi d \bar{\xi}}{\left(1+\frac{\Lambda}{6} \xi \bar{\xi}\right)^{2}}-2\left(\frac{1-\frac{\Lambda}{6} \xi \bar{\xi}}{1+\frac{\Lambda}{6} \xi \bar{\xi}}\right)^{2} d u d v+\left[\frac{\Lambda}{3}\left(\frac{1-\frac{\Lambda}{6} \xi \bar{\xi}}{1+\frac{\Lambda}{6} \xi \bar{\xi}}\right)^{2} v^{2}-\frac{1-\frac{\Lambda}{6} \xi \bar{\xi}}{1+\frac{\Lambda}{6} \xi \bar{\xi}} H\right] d u^{2} .
$$

Here $K N\left(\Lambda^{-}\right) I I$ means generalized Kundt waves 'of the second kind' with $\Lambda<0$. This class was first discovered by Ozsváth et.al. [3]. Observe that with $\kappa>0, \Lambda>0$, $K N\left(\Lambda^{+}\right) I I \equiv K N\left(\Lambda^{+}\right) I$, since transformation (5) where $A=\frac{1}{\sqrt{2}}, B=\sqrt{\frac{3}{\Lambda}}, a=1$, $\gamma=\delta=\Delta=0, u=\frac{\Lambda}{6} t$ identifies $K N\left(\Lambda^{+}\right)[\alpha=0, \beta=1]$ with $K N\left(\Lambda^{+}\right)\left[\alpha^{\prime}=1, \beta^{\prime}=0\right]$.

F. Subclass $\kappa=0, \Lambda<0$. The relation $\kappa=0$ implies $\alpha=1$. Thus, $|\beta|=\sqrt{-\frac{\Lambda}{6}}$ and the representative can be denoted by $K N\left(\Lambda^{-}\right) I I I \equiv K N(\Lambda<0)\left[\alpha=1, \beta=\sqrt{-\frac{\Lambda}{6}} e^{i \omega(u)}\right]$, $\omega(u)$ being an arbitrary real function of $u$. The metric takes the form of (四) with $q=$ $\left(1+\sqrt{-\frac{\Lambda}{6}} \xi e^{-i \omega(u)}\right)\left(1+\sqrt{-\frac{\Lambda}{6}} \bar{\xi} e^{i \omega(u)}\right)$. This class was also first discovered in [3]. One can distinguish two subsubclasses of $K N\left(\Lambda^{-}\right) I I I$ according to the value of $L: 1 . \quad L \neq 0(\mathbf{k}$ is not a Killing vector). 2. $L=0$ ( $\mathbf{k}$ is a Killing vector). In case $2 . \beta=$ const. and the transformation $\xi=\sqrt{\beta / \bar{\beta}} \eta$ leads to $K N\left(\Lambda^{-}\right) I I I_{K} \equiv K N(\Lambda<0)\left[\alpha=1, \beta=\sqrt{-\frac{\Lambda}{6}}\right]$, where the suffix ' $K$ ' stands for 'Killing':

$$
\begin{gathered}
K N\left(\Lambda^{-}\right) I I I_{K}: \quad d s^{2}=2 \frac{d \xi d \bar{\xi}}{\left(1+\frac{\Lambda}{6} \xi \bar{\xi}\right)^{2}}-2\left(\frac{\left(1+\sqrt{-\frac{\Lambda}{6}} \xi\right)\left(1+\sqrt{-\frac{\Lambda}{6}} \bar{\xi}\right)}{1+\frac{\Lambda}{6} \xi \bar{\xi}}\right)^{2} d u d v \\
-\frac{\left(1+\sqrt{-\frac{\Lambda}{6}} \xi\right)\left(1+\sqrt{-\frac{\Lambda}{6}} \bar{\xi}\right)}{1+\frac{\Lambda}{6} \xi \bar{\xi}} H d u^{2} .
\end{gathered}
$$

This subsubclass can be shown to be identical with the 'Lobatchevski waves' studied by Siklos [8]: indeed, the transformation $\xi=-\sqrt{-\frac{6}{\Lambda}}\left(x+\frac{1}{2}+i y\right) /\left(x-\frac{1}{2}+i y\right), v=\frac{12}{\Lambda} r$, brings the metric (14) into the form

$$
d s^{2}=-\frac{3}{\Lambda} \cdot \frac{1}{x^{2}}\left(d x^{2}+d y^{2}+2 d u d r+\tilde{H} d u^{2}\right)
$$


where $\tilde{H} \equiv-\frac{\Lambda}{6} x H$. This is the Siklos metric [8]. Recently we analyzed in detail the behaviour of test particles in these solutions and interpreted them as waves in the antide Sitter spacetime [10. Impulsive waves of this type were investigated in [11].

G. Relations of the subclasses. Using the above results we can summarize the invariant canonical classification of the $K N(\Lambda)$ class of solutions in the following diagram:

$$
K N(\Lambda)\left\{\begin{array}{l}
\Lambda=0\left\{\begin{array}{l}
\kappa=0: P P \\
\kappa>0: K N
\end{array}\right. \\
\Lambda \neq 0\left\{\begin{array}{l}
\kappa>0: K N(\Lambda) I \\
\kappa<0: K N\left(\Lambda^{-}\right) I I \\
\kappa=0: K N\left(\Lambda^{-}\right) I I I \rightarrow K N\left(\Lambda^{-}\right) I I I_{K} .
\end{array}\right.
\end{array}\right.
$$

There is an asymmetry with respect to the sign of $\Lambda$ : there are three distinct classes of non-expanding waves for $\Lambda<0$ whereas there is only one such class for $\Lambda>0$. The reason is in the condition $\kappa=\frac{\Lambda}{3} \alpha^{2}+2 \beta \bar{\beta}$ which for $\Lambda>0$ excludes the cases $\kappa<0$ and $\kappa=0$. Intuitively, fewer non-expanding waves 'fit' into the de Sitter universe which admits closed spacelike sections than into the anti-de Sitter space.

There exist natural relations between the $\Lambda=0$ and $\Lambda \neq 0$ subclasses. The metrics (12), (13) and (14) do not diverge as $\Lambda \rightarrow 0$, we can set $\Lambda=0$ and thus find

$$
K N(\Lambda=0) I=K N, \quad K N\left(\Lambda^{-}=0\right) I I=P P, \quad K N\left(\Lambda^{-}=0\right) I I I=P P .
$$

Thus, it is natural to consider the $K N(\Lambda) I$ class as a generalization of the Kundt solution $K N$, and the classes $K N\left(\Lambda^{-}\right) I I$ and $K N\left(\Lambda^{-}\right) I I I$ as generalizations of $P P$ waves. There exists no generalization of $P P$ waves to the case of $\Lambda>0$.

From metrics (10) and (15), with $\xi=(1 / \sqrt{2})(x+i y)$, and from (11) and (12) we find $d s_{K N\left(\Lambda^{-}\right) I I I_{K}}^{2}=-\frac{6}{\Lambda}(\xi+\bar{\xi})^{-2} d s_{P P}^{2}$, and $d s_{K N(\Lambda) I}^{2}=\left(1+\frac{\Lambda}{6} \xi \bar{\xi}\right)^{-2} d s_{K N}^{2}$. Therefore, the class $K N\left(\Lambda^{-}\right) I I I_{K}$ is conformal to the $P P$-class, and the class $K N(\Lambda) I$ is conformal to the $K N$-class. In fact, the solutions $K N\left(\Lambda^{-}\right) I I I_{K}$ and $K N(\Lambda) I$ are the only nontrivial space-times conformal to $P P$ and $K N$, respectively. The theorem proven by Siklos [8] states that the only vacuum solutions (other than PP solutions themselves) which are properly (with non-constant factor) conformal to non-flat $P P$ metrics are 
$K N\left(\Lambda^{-}\right) I I I_{K}$ metrics. However, we see from (16) that $P P$ metrics are a special case of metrics $K N\left(\Lambda^{-}\right) I I I_{K}$ for $\Lambda=0$, and Siklos' theorem may just be formulated as follows:

Proposition 1: The only vacuum solutions conformal to non-flat PP metrics are $K N\left(\Lambda^{-}\right) I I I_{K}$ metrics.

In addition, the following analogous proposition can be proven for the $K N$ solutions:

Proposition 2: The only vacuum solutions conformal to $K N$ metrics are $K N(\Lambda) I$ metrics.

The proof is contained in Appendix.

The conformal, homothetic, and isometric symmetries of the $K N(\Lambda)$ solutions have been systematically investigated by Salazar, García and Plebański [2] and by Siklos [8]. It is only in the subclasses $P P$ and $K N\left(\Lambda^{-}\right) I I I_{K}$ that the vector $\mathbf{k}=\partial_{v}$ is a Killing vector; $k_{\alpha ; \beta}=0$ only in the $P P$ subclass. Let us finally summarize the classification of all $K N(\Lambda)$ solutions and compare our notation with notations used in literature:

\begin{tabular}{|l|l|}
\hline Notation in this paper & Notations in literature \\
\hline$K N(\Lambda)$ & $R(\Lambda, \alpha, \beta)$ [3] \\
\hline$P P$ & $p p, R$ [4], [3] for Refs. \\
\hline$K N$ & $K$ [6], [四] \\
\hline$K N(\Lambda) I$ & $K(\Lambda)$ []], [3] \\
\hline$K N\left(\Lambda^{-}\right) I I$ & $R(\Lambda)$ [3] \\
\hline$K N\left(\Lambda^{-}\right) I I I$ & $(I V)_{1}$ [3] \\
\hline$K N\left(\Lambda^{-}\right) I I I_{K}$ & $(I V)_{0}$ [ [], [3] \\
\hline
\end{tabular}

Impulsive waves in the $K N(\Lambda)$ spacetimes were recently studied in [12].

\section{THE ROBINSON-TRAUTMAN CLASS OF SOLUTIONS $R T N(\Lambda, \epsilon)$}

The Robinson-Trautman solutions [7] satisfying the vacuum equations with $\Lambda$ can be written as (see 四)

$$
d s^{2}=2 \frac{r^{2}}{P^{2}} d \zeta d \bar{\zeta}-2 d u d r-\left[\Delta \ln P-2 r(\ln P)_{, u}-\frac{2 m}{r}-\frac{\Lambda}{3} r^{2}\right] d u^{2},
$$


where $\zeta$ is a complex spatial coordinate, $r$ is an affine parameter along the rays generated by the vector field $\mathbf{k}, u$ is a retarded time, $m$ is a function of $u$ which in some cases can be interpreted as mass, and $\Delta \equiv 2 P^{2} \partial^{2} / \partial \zeta \partial \bar{\zeta}$. The function $P \equiv P(\zeta, \bar{\zeta}, u)$ satisfies the equation $\Delta \Delta(\ln P)+12 m(\ln P)_{, u}-4 m_{, u}=0$. Here we restrict attention to the solutions of type $N$ and denote these as $R T N(\Lambda)$. In this case $m=0$ and $\Delta \ln P=K(u)$. By a transformation $u=g(\tilde{u}), r=\tilde{r} / \dot{g}$, where $\dot{g}=d g / d \tilde{u}$, we can set the Gaussian curvature $K(u)$ of the 2 -surfaces $2 P^{-2} d \zeta d \bar{\zeta}$ to be $K=2 \epsilon$, where $\epsilon=+1,0,-1$ (since $\tilde{P}=\dot{g} P$ and $\tilde{K}=\dot{g}^{2} K$, the sign of $K$ is invariant). Thus, the different subclasses can be denoted as $\operatorname{RTN}(\Lambda, \epsilon)$. The corresponding metrics can be written as

$$
d s^{2}=2 \frac{r^{2}}{P^{2}} d \zeta d \bar{\zeta}-2 d u d r-2\left[\epsilon-r(\ln P)_{, u}-\frac{\Lambda}{6} r^{2}\right] d u^{2}
$$

Since $\epsilon=+1,0,-1$ and $\Lambda>0, \Lambda=0, \Lambda<0$, there are 9 invariant subclasses.

Another coordinates for the $R T N(\Lambda, \epsilon)$ class, suitable for physical interpretation, has been given by García Díaz and Plebański [1]. Their metric is expressed in terms of a function $f(\xi, u)$ which is an arbitrary function of $u$, analytic in spatial coordinate $\xi$

$$
d s^{2}=2 v^{2} d \xi d \bar{\xi}+2 v \overline{\mathrm{A}} d \xi d u+2 v \mathrm{~A} d \bar{\xi} d u+2 \psi d u d v+2(\mathrm{~A} \overline{\mathrm{A}}+\psi \mathrm{B}) d u^{2},
$$

where

$$
\mathrm{A}=\epsilon \xi-v f, \quad \mathrm{~B}=-\epsilon+\frac{v}{2}\left(f_{, \xi}+\bar{f}_{, \bar{\xi}}\right)+\frac{\Lambda}{6} v^{2} \psi, \quad \psi=1+\epsilon \xi \bar{\xi}
$$

It can be shown that the transformation relating (18) with $(19)$ has the form

$$
\begin{aligned}
& \xi=F(\zeta, u)=\int f(\xi(\zeta, u), u) d u \\
& v=\frac{r}{1+\epsilon F \bar{F}}, \\
& u \rightarrow-u, \quad P=(1+\epsilon F \bar{F})\left(F_{, \zeta} \bar{F}_{, \bar{\zeta}}\right)^{-\frac{1}{2}} .
\end{aligned}
$$

If $f$ does not depend on $\xi$, we put $\xi=F(\zeta, u)=\zeta+\int f(u) d u$.

The non-vanishing Weyl tensor components are proportional to $f_{, \xi \xi \xi}$ so that the solutions are conformally flat if $f$ is quadratic in $\xi$. Thus, we can formulate

Lemma 3: The $R T N(\Lambda, \epsilon)$ solutions (19) with $f=f_{c}=c_{0}(u)+c_{1}(u) \xi+c_{2}(u) \xi^{2}$, where $c_{i}(u)$ are arbitrary complex functions of $u$, are isometric to Minkowski (if $\Lambda=0$ ), de Sitter $(\Lambda>0)$ and anti-de Sitter spacetime $(\Lambda<0)$. 
Transformations preserving the form of (19) were studied in [2]. However, more general transformations (Eq. (2.4) in [2] follows from Eqs. (21), (24) if we put $\Delta=0$ and $\alpha \bar{\alpha}+\epsilon \beta \bar{\beta}=1$ ) are given in the following

Lemma 4: The coordinate transformations $(v, \xi, \bar{\xi}, u) \rightarrow(w, \eta, \bar{\eta}, t)$, which maintain invariant the form of the $R T N(\Lambda, \epsilon)$ metric (19), are

$$
\begin{aligned}
\xi & =\frac{\alpha \eta+\beta}{\gamma \eta+\delta}, \\
v & =\frac{(\gamma \eta+\delta)(\bar{\gamma} \bar{\eta}+\bar{\delta})}{\sqrt{(\alpha \delta-\beta \gamma)(\bar{\alpha} \bar{\delta}-\bar{\beta} \bar{\gamma})}} w, \\
u & =\int \frac{\sqrt{(\alpha \delta-\beta \gamma)(\bar{\alpha} \bar{\delta}-\bar{\beta} \bar{\gamma})}}{\delta \bar{\delta}+\epsilon \beta \bar{\beta}} d t,
\end{aligned}
$$

where $\alpha(t), \beta(t), \gamma(t), \delta(t)$ are arbitrary complex functions of $t$ which satisfy the conditions

$$
\bar{\gamma} \delta=-\epsilon \bar{\alpha} \beta, \quad \gamma \bar{\gamma}+\epsilon \alpha \bar{\alpha}=\epsilon(\delta \bar{\delta}+\epsilon \beta \bar{\beta}) .
$$

In the coordinates $(w, \eta, \bar{\eta}, t)$, new structural function $f^{\prime}(\eta, t)$ is related to $f$ as follows:

$$
\begin{aligned}
f^{\prime}= & \frac{\bar{\alpha} \bar{\delta}-\bar{\beta} \bar{\gamma}}{\delta \bar{\delta}+\epsilon \beta \bar{\beta}} \frac{(\gamma \eta+\delta)^{2}}{\sqrt{(\alpha \delta-\beta \gamma)(\bar{\alpha} \bar{\delta}-\bar{\beta} \bar{\gamma})}} f \\
& -\frac{1}{\alpha \delta-\beta \gamma}\left[(\dot{\beta} \delta-\beta \dot{\delta})+(\dot{\alpha} \delta-\alpha \dot{\delta}+\dot{\beta} \gamma-\beta \dot{\gamma}) \eta+(\dot{\alpha} \gamma-\alpha \dot{\gamma}) \eta^{2}\right] .
\end{aligned}
$$

From this relation and Lemma 3 we see that the conformally flat part $f_{c}$ is indeed unimportant since it can be generated from $f=0$ by the coordinate transformation (21).

If $\epsilon \neq 0$, Eq. (22) implies that $|\alpha|=|\delta|,|\beta|=|\gamma|$ so that $\sqrt{(\alpha \delta-\beta \gamma)(\bar{\alpha} \bar{\delta}-\bar{\beta} \bar{\gamma})}=$ $\delta \bar{\delta}+\epsilon \beta \bar{\beta}$. Equations (21)-(23) then simplify to

$$
\xi=\frac{\alpha \eta+\beta}{\gamma \eta+\delta}, \quad v=\frac{(\gamma \eta+\delta)(\bar{\gamma} \bar{\eta}+\bar{\delta})}{\alpha \bar{\alpha}+\epsilon \beta \bar{\beta}} w, \quad u=t
$$

where $\alpha(t)$ and $\beta(t)$ are arbitrary complex functions of $t$ and $\gamma(t)=\bar{\beta}(t) e^{i \Gamma(t)}, \delta(t)=$ $\bar{\alpha}(t) e^{i \Delta(t)}$, with $\Gamma(t), \Delta(t)$ being arbitrary real functions of $t$ satisfying $\Delta-\Gamma=(1+\epsilon) \frac{\pi}{2}$. The structural function is given by $f^{\prime}=\left\{(\gamma \eta+\delta)^{2} f-[(\dot{\beta} \delta-\beta \dot{\delta})+(\dot{\alpha} \delta-\alpha \dot{\delta}+\dot{\beta} \gamma-\right.$ $\left.\left.\beta \dot{\gamma}) \eta+(\dot{\alpha} \gamma-\alpha \dot{\gamma}) \eta^{2}\right]\right\} e^{-i \Delta} /(\alpha \bar{\alpha}+\epsilon \beta \bar{\beta})$

For $\epsilon=0$, equations (22) imply $\gamma=0$ so that the transformations (21) yield

$$
\xi=A(t) \eta+B(t), \quad v=\frac{w}{\sqrt{A(t) \bar{A}(t)}}, \quad u=\int \sqrt{A(t) \bar{A}(t)} d t
$$


where $A(t)$ and $B(t)$ are arbitrary complex functions of $t$. The relation (23) between the structural functions is now $f^{\prime}=\sqrt{\bar{A} / A} f-(\dot{B} / A+\dot{A} / A \eta)$, so that, in contrast to the case $\epsilon \neq 0$, the term quadratic in $\eta$ vanishes. Hence, Eq. (25) does not enable us to transform away the complete conformally flat part $f_{c}$. (We tried but without success to generalize (25) so that quadratic terms could be removed.)

Symmetries of the $\operatorname{RTN}(\Lambda, \epsilon)$ solutions have been investigated in [13] (see also [4], Table 33.2) and, more systematically, in [2]. The solutions which are not conformally flat allow the existence of at most two Killing vectors.

\section{ACKNOWLEDGMENTS}

We thank Jerry Griffiths for reading the manuscript and useful suggestions. We also acknowledge the support of grant GACR-202/99/0261 from the Czech Republic.

\section{APPENDIX: PROOF OF PROPOSITION 2}

For the conformally related metrics $g_{\alpha \beta}$ and $\hat{g}_{\alpha \beta}, \hat{g}_{\alpha \beta}=\Omega^{-2} g_{\alpha \beta}$, the trace-free Ricci tensors are related by [4]

$$
\begin{aligned}
& S_{\alpha \beta}=R_{\alpha \beta}-\frac{1}{4} R g_{\alpha \beta}, \\
& \hat{S}_{\alpha \beta}=\hat{R}_{\alpha \beta}-\frac{1}{4} \hat{R} \hat{g}_{\alpha \beta}=S_{\alpha \beta}+\frac{2}{\Omega}\left(\Omega_{; \alpha \beta}-\frac{1}{4} g_{\alpha \beta} \square \Omega\right),
\end{aligned}
$$

where $\square \Omega=g^{\mu \nu} \Omega_{; \mu \nu}$, the covariant derivative is taken with respect to $g_{\alpha \beta}$, and the scalar curvatures is

$$
\hat{R}=\Omega^{2} R+6 \Omega \square \Omega-12 g^{\alpha \beta} \Omega_{; \alpha} \Omega_{; \beta}
$$

Since $\hat{R}_{\alpha \beta}=\Lambda \hat{g}_{\alpha \beta}, \hat{R}=4 \Lambda$, we have $\hat{S}_{\alpha \beta}=0$. In coordinates $(v, \xi, \bar{\xi}, u)$,

$$
g_{12}=1, \quad g_{03}=-(\xi+\bar{\xi})^{2}, \quad g_{33}=2(\xi+\bar{\xi})^{2}\left(v^{2}-\frac{1}{2} \frac{H}{\xi+\bar{\xi}}\right)
$$

$R_{33}=(\xi+\bar{\xi}) H_{, \xi \bar{\xi}}$, and the other Ricci tensor components vanish. Therefore, $R=0$ and (26) can be written as

$$
\Omega_{, \alpha \beta}-\Gamma_{\alpha \beta}^{\gamma} \Omega_{, \gamma}-\frac{1}{4} g_{\alpha \beta} \square \Omega+\frac{1}{2} \Omega R_{\alpha \beta}=0
$$


which gives

$$
\begin{aligned}
& \left(\frac{\Omega_{, v}}{\xi+\bar{\xi}}\right)_{, \xi}=0=\left(\frac{\Omega_{, v}}{\xi+\bar{\xi}}\right)_{, \bar{\xi}}, \\
& \Omega_{, v v}=0, \\
& \Omega_{, \xi \xi}=0=\Omega_{, \bar{\xi} \bar{\xi}} \\
& \Omega_{, v u}+2 v \Omega_{, v}-(\xi+\bar{\xi})\left(\Omega_{, \xi}+\Omega_{, \bar{\xi}}\right)+\frac{1}{4}(\xi+\bar{\xi})^{2} \square \Omega=0 \\
& \Omega_{, \xi \bar{\xi}}=\frac{1}{4} \square \Omega, \\
& \Omega_{, \xi u}-\frac{\Omega_{, u}}{\xi+\bar{\xi}}-\frac{1}{2}\left(\frac{H}{\xi+\bar{\xi}}\right)_{, \xi} \Omega_{, v}=0=\Omega_{, \bar{\xi} u}-\frac{\Omega_{, u}}{\xi+\bar{\xi}}-\frac{1}{2}\left(\frac{H}{\xi+\bar{\xi}}\right)_{, \bar{\xi}} \Omega_{, v}, \\
& \Omega_{, u u}-2 v \Omega_{, u}-\left[2 v\left(2 v^{2}-\frac{H}{\xi+\bar{\xi}}\right)+\frac{1}{2}\left(\frac{H}{\xi+\bar{\xi}}\right)_{, u}\right] \Omega_{, v} \\
& \quad-\frac{1}{2}[(\xi+\bar{\xi}) H]_{, \bar{\xi}} \Omega_{, \xi}-\frac{1}{2}[(\xi+\bar{\xi}) H]_{, \xi} \Omega_{, \bar{\xi}}+2 v^{2}(\xi+\bar{\xi})\left(\Omega_{, \xi}+\Omega_{, \bar{\xi}}\right) \\
& \quad-\frac{1}{2}(\xi+\bar{\xi})^{2}\left(v^{2}-\frac{1}{2} \frac{H}{\xi+\bar{\xi}}\right) \square \Omega+\frac{1}{2}(\xi+\bar{\xi}) H_{, \xi \bar{\xi}} \Omega=0 .
\end{aligned}
$$

By using (34), Eq. (27) takes the form

$$
\Omega_{, \xi \bar{\xi}}-\Omega_{, \xi} \Omega_{, \bar{\xi}}+\frac{\Omega_{, v}}{(\xi+\bar{\xi})^{2}}\left[\Omega_{, u}+\left(v^{2}-\frac{1}{2} \frac{H}{\xi+\bar{\xi}}\right) \Omega_{, v}\right]=\frac{\Lambda}{6} .
$$

Eqs. (30) imply $\Omega_{, v}=A(u, v)(\xi+\bar{\xi})$. Eq. (31) gives $A_{, v}=0$ so that $\Omega=A(u)(\xi+\bar{\xi}) v+$ $B(\xi, \bar{\xi}, u)$. By virtue of Eq. (32) it must be of the form $\Omega=A(u)(\xi+\bar{\xi}) v+C(u) \xi \bar{\xi}+$ $D_{1}(u) \xi+D_{2}(u) \bar{\xi}+E(u)$. Eq. (33) combined with Eq. (34) gives $d A / d u=D_{1}+D_{2}$ so that $\Omega=A(u)(\xi+\bar{\xi}) v+C(u) \xi \bar{\xi}+\frac{1}{2} d A / d u(\xi+\bar{\xi})+D(u)(\xi-\bar{\xi})+E(u)$ with $A, C, E$ being real functions of $u$, and $D(u)$ being pure imaginary. We distinguish the possibilities:

$$
\text { 1) } A=0
$$

In this case Eq. (35) implies $\Omega=C \xi \bar{\xi}+D(\xi-\bar{\xi})+E$, with $C, E$ real constants, $D$ a pure imaginary constant. Let $(\mathrm{i}) C \neq 0$. Without loss of generality we can set $D=0$ by transformation $\xi \rightarrow \xi^{\prime}=\xi-D / C$. If $E \neq 0$, we can set $E=1$ by $\xi^{\prime}=\xi / E$ which implies $\Omega=C \xi \bar{\xi}+1$. Eq. (37) gives $C=\Lambda / 6$ so that $\Omega=1+(\Lambda / 6) \xi \bar{\xi}$, and the metric $\hat{g}_{\alpha \beta}$ takes the canonical form (12) of the $K N(\Lambda) I$ metric. If $E=0$ we can make transformation $\xi^{\prime}=1 /(C \xi)$, after which $\hat{g}_{\alpha \beta}$ takes the canonical form (11) of the $K N$ metric. Now (ii) $C=0$. If $D \neq 0$ we can set $E=0$ by $\xi^{\prime}=\xi+E /(2 D)$. The relation 
(37) gives $\Omega=\sqrt{\Lambda / 6}(\xi-\bar{\xi})$, with $\Lambda$ necessarily being negative. It represents just another coordinate form of the $K N(\Lambda) I$ metric since by $\xi=\left(\xi^{\prime}+i \sqrt{-6 / \Lambda}\right) /\left(i \xi^{\prime}+\sqrt{-6 / \Lambda}\right)$ we get the $K N\left(\Lambda^{-}\right) I$ metric in the canonical form (12). If $D=0$ then $\Omega=E$ so that $\hat{g}_{\alpha \beta}$ is the $K N$ metric by transformation $\xi^{\prime}=|E| \xi$.

$$
\text { 2) } A \neq 0
$$

Without loss of generality we can assume $A=1$ by using transformation $w=A(u) v+$ $\frac{1}{2} d A / d u, t=\int A^{-1}(u) d u$. Therefore, $\Omega=(\xi+\bar{\xi}) v+C(u) \xi \bar{\xi}+D(u)(\xi-\bar{\xi})+E(u)$. In this case Eq. (35) implies $H=2\left[\frac{d C}{d u} \xi \bar{\xi}+\alpha(u)(\xi+\bar{\xi})+\frac{d D}{d u}(\xi-\bar{\xi})+\frac{d E}{d u}\right]$, with $\alpha$ being an arbitrary real function of $u$. Eq. (37) reduces to $\alpha(u)=C(u) E(u)+D(u)^{2}-\Lambda / 6$, whereas Eq. (36) is satisfied identically. The solution given by $\hat{g}_{\alpha \beta}$ is then conformally flat since $g_{\alpha \beta}$ for $H$ of this form is conformally flat $\left(C_{\alpha \beta \gamma \delta}=0\right)$. Therefore, the solution describes Minkowski, de Sitter or anti-de Sitter spacetime, according to the sign of $\Lambda$. It is interesting to notice that although $\hat{g}_{\alpha \beta}$ of this form describes a conformally flat vacuum solution, the $K N$ solution to which it is conformal (given by $g_{\alpha \beta}$ with $H$ of the same form) is conformally flat but not necessarily a vacuum solution. In general, it is a pure radiation solution, becoming a vacuum solution (Minkowski) only for $C=$ const. In particular, if $\alpha=0$ and $D, E=$ const., then the conformally flat $K N$ pure radiation solution given by $H=2 \frac{d C}{d u} \xi \bar{\xi}$ is Wils' solution (3.10) [14] for $N=0$ (where $\left.Q+\bar{Q}=\frac{d C}{d u}, v \rightarrow v /(\xi+\bar{\xi})^{2}\right)$. The solution was used by Wils as an explicit counterexample of theorem 32.17 in [4] according to which there are no other conformally flat pure radiation solutions besides the special PP wave of McLenaghan et al. 15.

We have thus shown that all vacuum solutions conformal to the $K N$ class are 1 ) $K N(\Lambda) I$ solutions, 2) $K N$ solutions themselves $(K N=K N(\Lambda=0) I), 3)$ Minkowski, de Sitter and anti-de Sitter space-times (these are special cases of $K N(\Lambda) I$ given by $\left.K N(\Lambda) I\left[H=H_{c}\right]\right)$. Therefore, all vacuum solutions conformal to the $K N$ class belong to the $K N(\Lambda) I$ class, which proves Proposition 2 in Section II.

\section{References}


[1] A. García Díaz and J. F. Plebański, "All nontwisting N's with cosmological constant," J. Math. Phys. 22, 2655 (1981).

[2] H. I. Salazar, A. García Díaz, and J. F. Plebański, "Symmetries of the nontwisting type-N solutions with cosmological constant," J. Math. Phys. 24, 2191 (1983).

[3] I. Ozsváth, I. Robinson, and K. Rózga, 'Plane-fronted gravitational and electromagnetic waves in spaces with cosmological constant," J. Math. Phys. 26, 1755 (1985).

[4] D. Kramer, H. Stephani, M. A. H. MacCallum, and H. Herlt, Exact Solutions of the Einstein's Field Equations (Cambridge University Press, Cambridge, 1980).

[5] W. Kundt and A. Thompson, "Le tenseur de Weyl et une congruence associée de géodésiques isotropes sans distortion," C.R.Acad.Sci. (Paris) 254, 4257 (1962).

[6] W. Kundt, "The Plane-fronted Gravitational Waves," Z. Phys. 163, 77 (1961).

[7] I. Robinson and A. Trautman, "Spherical gravitational waves," Phys. Rev. Lett. 4, 431 (1960).

[8] S. T. C. Siklos, "Lobatchevski Plane Gravitational Waves," in Galaxies, Axisymmetric Systems and Relativity, edited by M. A. H. MacCallum (Cambridge University Press, Cambridge, 1985).

[9] A. García Díaz, "The Generalized Kundt Solution with Cosmological Constant," Nuovo Cim. 78B, 255 (1983).

[10] J. Podolský, "Interpretation of the Siklos solutions as exact gravitational waves in the anti-de Sitter universe," Class. Quantum Grav. 15, 719 (1998).

[11] J. Podolský and J. B. Griffiths "Impulsive waves in de Sitter and anti-de Sitter space-times generated by null particles with an arbitrary multipole structure," Class. Quantum Grav. 15, 453 (1998).

[12] J. Podolský, "Non-expanding impulsive gravitational waves," Class. Quantum Grav. 15, 3229 (1998).

[13] C. D. Collinson and D. C. French, "Null tetrad approach to motions in empty spacetime," J. Math. Phys. 8, 701 (1967).

[14] P. Wils, "Homogeneous and conformally Ricci flat pure radiation fields," Class. Quantum Grav. 6, 1243 (1989). 
[15] R. G. McLenaghan, N. Tariq, and B. J. O. Tupper, "Conformally flat solutions of the Einstein-Maxwell equations for null electromagnetic fields," J.Math. Phys. 16, 829 (1975). 